\title{
LYPD8 regulates the proliferation and migration of colorectal cancer cells through inhibiting the secretion of IL-6 and TNF- $\alpha$
}

\author{
JIANBIN XU $^{1 *}$, JUN QIAN $^{2 *}$, WEI ZHANG ${ }^{1}$, ENGENG CHEN $^{1}$, GUOLIN ZHANG $^{1}$, \\ GAOYANG CAO $^{1}$, FEI WANG ${ }^{1}$, XUNING SHEN ${ }^{1}$, \\ WEI ZHOU ${ }^{1}$ and ZHANGFA SONG ${ }^{1}$
}

\author{
${ }^{1}$ Department of Colorectal Surgery, Sir Run Run Shaw Hospital, School of Medicine, Zhejiang University, \\ Hangzhou, Zhejiang 310016; ${ }^{2}$ Department of Colorectal Surgery, Xinchang People's Hospital, \\ Xinchang, Zhejiang 312500, P.R. China
}

Received September 12, 2018; Accepted February 15, 2019

DOI: $10.3892 / o r .2019 .7034$

\begin{abstract}
Ly6/Plaur domain-containing 8 (LYPD8) contributes to the segregation of intestinal microbiota and intestinal epithelia and is critical for the prevention of intestinal inflammation. However, its relevance in cancer biology remains to be fully elucidated. The present study aimed to clarify the biological effects of LYPD8 on colon cancer tissue from patients and colorectal cancer (CRC) cells. The results revealed that the expression of LYPD8 was significantly reduced in the CRC tissue compared with that in precancerous tissue and normal tissue, particularly in stage III tissue. The results also revealed increased levels of P65 and signal transducer and activator of transcription 3 (STAT3) phosphorylation and increased secretion of interleukin-6 (IL-6) and tumor necrosis factor- $\alpha$ (TNF- $\alpha$ ) in CRC tissue compared with levels in precancerous tissue. Supporting these findings, the levels of secreted TNF- $\alpha$ and IL- 6 were significantly reduced when LYPD8 was overexpressed in human CRC cells, and the secretion of TNF- $\alpha$ and IL- 6 were positively associated with the phosphorylation of STAT3 and P65. However, this trend was restored upon supplementation with TNF- $\alpha$ and IL-6 in CRC cells. Furthermore, the overexpression of LYPD8 in CRC cells significantly inhibited CRC cell proliferation and migration. Overall, the LYPD8-mediated tumor-inhibiting role involves a
\end{abstract}

Correspondence to: Professor Zhangfa Song or Dr Wei Zhou, Department of Colorectal Surgery, Sir Run Run Shaw Hospital, School of Medicine, Zhejiang University, 3 Qingchun Road, Hangzhou, Zhejiang 310016, P.R. China

E-mail: songzhangfa@zju.edu.cn

E-mail: weizhou_srrsh@zju.edu.cn

*Contributed equally

Key words: Ly6/Plaur domain-containing 8, colorectal cancer, interleukin- 6 , tumor necrosis factor- $\alpha$ direct effect on the secretion of IL-6/TNF- $\alpha$ in CRC cells by reducing the phosphorylation of STAT3 and P65.

\section{Introduction}

Recently, a highly glycosylated glycosylphosphatidylinositolanchored protein, known as Ly6/Plaur domain-containing 8 (LYPD8), was identified as a novel molecule contributing to the segregation of intestinal bacteria and intestinal epithelia in the large intestine (1). Lypd8, which is anchored to intestinal epithelial cells in the uppermost epithelial layer, is constitutively shed into the intestinal lumen and preferentially binds to flagellated bacteria from various genera (2). Mice lacking LYPD8 lack the bacteria-free space immediately above the epithelial layer of the colon, indicating that LYPD8 is critical for the segregation of intestinal bacteria and colonic epithelia (3). Furthermore, LYPD8 is also expressed in the colonic epithelia, and the expression of LYPD8 is reduced in the colon of some patients with ulcerative colitis (UC) (4). However, the biological action and functions of LYPD8 in colorectal cancer (CRC) remain to be fully elucidated.

Previous studies have confirmed that several cytokines are involved in the transition from inflammation to tumor (5). Interleukin-6 (IL-6) is an inflammatory factor with a wide range of biological effects, including tumor-promoting effects (6). The levels of IL-6 have been observed to be significantly increased in the serum of patients with $\mathrm{UC}$, and the degree of the increase was associated with the disease (7). The expression of IL-6 is regulated by nuclear factor- $\kappa \mathrm{B}(\mathrm{NF}-\kappa \mathrm{B})$, which is expressed in multiple cells and is involved in regulating the transcription of tumor proliferation-related genes (8). NF- $\kappa \mathrm{B}$ is typically present as a dimer or heterodimer, such as the P65/P50 heterodimer, in cells (9). Tumor necrosis factor- $\alpha(\mathrm{TNF}-\alpha)$ is another important inflammatory factor, and increased TNF- $\alpha$ expression is present in serum and colon mucosa of patients with CRC (10). TNF- $\alpha$ promotes the progression of CRC, and its mechanism may be associated with activation of the NF- $\kappa \mathrm{B}$ signaling pathway (11). 
The signal transducer and activator of transcription 3 (STAT3) signaling pathway acts as a bridge between inflammation and tumors (12). This signaling pathway is a positive feedback regulator in various types of cancer, including CRC, prostate, lung, breast, pancreatic and ovarian cancer (13). The activation of STAT3 can induce the abnormal transcription of oncogenes associated with cell proliferation, apoptosis and differentiation (14). Numerous cytokines, including IL-6 and TNF- $\alpha$, exert biological effects in cancer cells through the STAT3 signaling pathway (15). Previous studies have demonstrated that the overexpression of IL-6 can increase the anti-apoptotic effect in cancer cells through the IL-6-STAT3 signaling pathway (16).

In the present study, to evaluate the effects of LYPD8 in the biological function of cancer, the expression of LYPD8 was examined, and the corresponding levels of STAT3/P65 phosphorylation in and secretion of IL-6/TNF- $\alpha$ from CRC tissues at different tumor stages were compared with corresponding precancerous tissue and normal tissue. Following this, IL-6/TNF- $\alpha$ secretion, STAT3/P65 dephosphorylation and cell proliferation and migration were detected when LYPD8 was overexpressed in CRC cells.

\section{Materials and methods}

Histological analysis. A total of 40 patients with CRC (range, 45-70 years old) who underwent surgery between 2014 and 2015 at Sir Run Run Shaw Hospital (Hangzhou, China) were recruited. All experiments using human tissues were approved by the local institutional Ethics Committee (institutional review board reference no. 20140213-19). Written informed consent was obtained from patients prior to their inclusion in the study. The histological types, disease stages and cancer cell contents in each formalin-fixed paraffin-embedded section were examined by experienced pathologists. Normal tissue samples ( $>3 \mathrm{~cm}$ from the edge of cancer tissues) and precancerous tissue samples (within $3 \mathrm{~cm}$ from the edge of cancer tissues) were used as controls. None of the patients included in the present study had received preoperative radiotherapy, chemotherapy or immunotherapy prior to surgery. The tissues were fixed overnight in $4 \%$ paraformaldehyde solution, dehydrated and embedded in paraffin. The tissue sections were then deparaffinized in xylene and rehydrated in graded ethanol, followed by antigen microwave retrieval. The sections were blocked with $3 \% \mathrm{H}_{2} \mathrm{O}_{2}$ and $10 \%$ goat serum (cat. no. ab7481; Abcam, Cambridge, UK), and then incubated overnight at $4^{\circ} \mathrm{C}$ with STAT3 antibody (dilution 1:100; cat. no. ab68153; Abcam) and P65 antibody (dilution 1:100; cat. no. ab32536; Abcam). The sections were then incubated at $37^{\circ} \mathrm{C}$ for $1 \mathrm{~h}$ with FITC/PE-conjugated antibody (dilution 1:200; cat. no. A0562/A0423; Beyotime Institute of Biotechnology, Haimen, China). The nuclei were stained DAPI (Beyotime Institute of Biotechnology) for $5 \mathrm{~min}$. All sections were observed under a confocal laser microscope (LSM780; Carl Zeiss AG, Oberkochen, Germany).

Western blotting. The preparation of protein extracts from the tissues/cells and electrophoretic separation were performed as previously described (17). Following electrophoresis, the proteins were transferred onto a polyvinylidene difluoride (PVDF) membrane (Merck KGaA, Darmstadt, Germany), which was blocked in 5\% non-fat milk (for $1 \mathrm{~h}$ ) and further incubated with a STAT3 antibody (dilution 1:1,000; cat. no. ab68153; Abcam), phosphorylated (p)-STAT3 antibody (dilution 1:1,000; cat. no. AF3294; Affinity Biosciences, Zhenjiang, China), P65 antibody (dilution 1:1,000; cat. no. ab32536; Abcam), p-P65 antibody (dilution 1:1,000; cat. no. AF2006; Affinity Biosciences) and GAPDH antibody (dilution 1:2,000; cat. no. AP0063; Bioworld Technology, Inc., St. Louis Park, MN, USA) overnight at $4^{\circ} \mathrm{C}$. Following incubation, the blots were incubated with the appropriate alkaline phosphatase-labeled secondary antibody (dilution 1:5,000; cat. no. AF1030; Beyotime Institute of Biotechnology) for $2 \mathrm{~h}$ at room temperature. Following stringent washing with Tris-buffered saline (TBS) and Tween-20, the membranes were incubated with the alkaline phosphatase substrate for $5 \mathrm{~min}$ at room temperature and then submitted to fluorescence detection using the VersaDoc 3000 Imaging system (Bio-Rad Laboratories, Inc., Hercules, CA, USA). The band intensities were analyzed using ImageJ v1.8.0 software (NIH; National Institutes of Health, Bethesda, MD, USA).

ELISA. The human IL-6 ELISA kit (cat. no. HM10205; Bio-Swamp Life Science, Shanghai, China) and human TNF- $\alpha$ ELISA kit (cat. no. HM10001; Bio-Swamp Life Science) were used for the quantitation of IL- 6 and TNF- $\alpha$, respectively. The procedure was performed according to the manufacturer's protocol.

Isolation of RNA and reverse transcription-quantitative polymerase chain reaction (RT-qPCR) analysis. Total RNA was extracted from the three cell lines using a Takara MiniBEST Universal RNA Extraction kit (Takara Biotechnology Co.,Ltd., Dalian, China) according to the manufacturer's protocol and quantified with a NanoDrop spectrophotometer. Total RNA was reverse transcribed with the PrimeScript ${ }^{\circledR}$ RT reagent kit (Takara Biotechnology Co., Ltd.) and random hexamers in a volume of $20 \mu \mathrm{l}$ according to the manufacturer's protocol. Then samples (4 samples for each group) performed out in a PCR gene amplifcation apparatus: $42^{\circ} \mathrm{C}$ for $60 \mathrm{~min}, 70^{\circ} \mathrm{C}$ for $5 \mathrm{~min}$, and then the reaction was terminated, placed on ice for storage or stored at $-20^{\circ} \mathrm{C}$. RT-qPCR was performed with z qPCR kit (SYBR Premix Ex Taq; Takara Biotechnology Co., Ltd.) using an Applied Bio-Rad CFX96 Sequence Detection system. The following primers were used for RT-PCR: LYPD8, forward 5'-CGAAAGTTTGAGTGTGCAAATGT-3' and reverse 5'-CAGAGCAGGGAAAGAGGGTGT-3'; $\beta$-actin, forward 5'-TGACGTGGACATCCGCAAAG-3' and reverse 5'-CTGGAAGGTGGACAGCGAGG-3'. The qPCR thermocycling conditions were performed as follows: Pre-deformation $95^{\circ} \mathrm{C}$ for $10 \mathrm{~min}, 1$ cycle; denaturation $95^{\circ} \mathrm{C}$ for $15 \mathrm{sec}$ and annealing $60^{\circ} \mathrm{C}$ for $60 \mathrm{sec}, 40$ cycles. All reactions were normalized to the housekeeping gene $\beta$-actin to quantify the relative gene expression and were then analyzed using the $2^{-\Delta \Delta \mathrm{Cq}}$ method (18).

Cell culture. Three CRC cell lines (SW480, HT29 and SW620) were used. SW480 (ATCC ${ }^{\circledast}$ CCL-228 ${ }^{\mathrm{TM}}$, organism, human; tissue, colon; disease, colorectal adenocarcinoma), HT29 (ATCC ${ }^{\circledR}$ HTB-38 ${ }^{\mathrm{TM}}$, organism, human; tissue, colon; disease, 


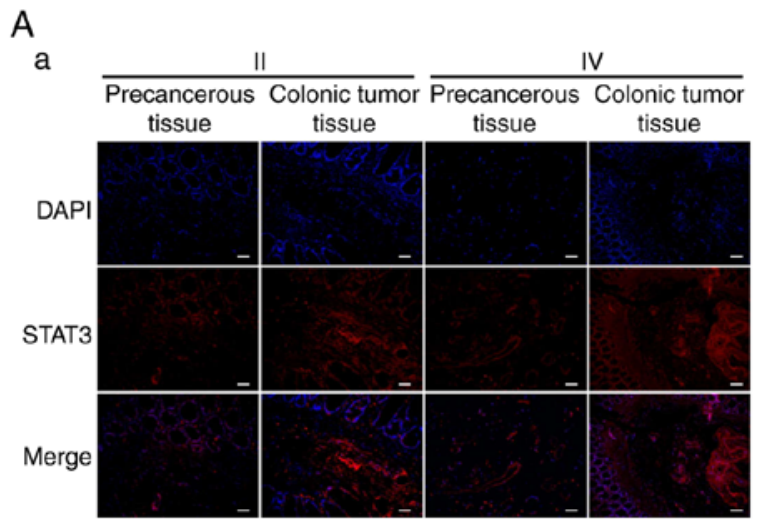

B

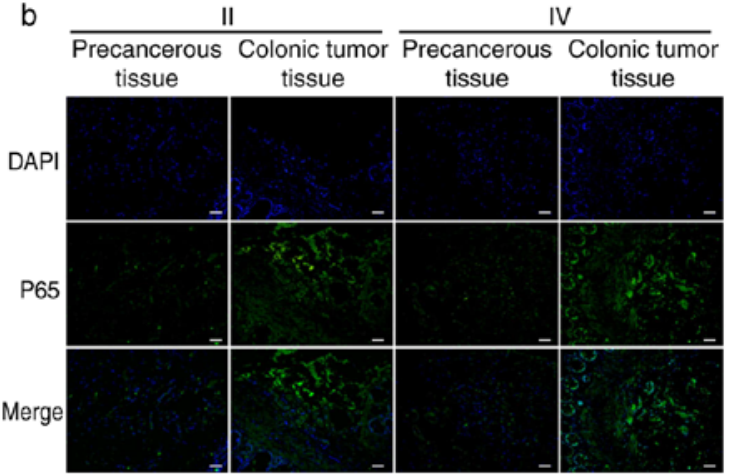

C Phosphorylation levels of P65 and STAT3

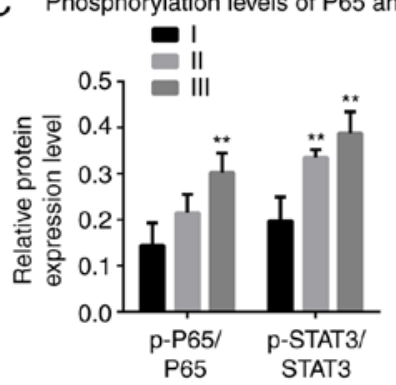

Figure 1. STAT3 and P65 are activated in colonic tumor tissues from patients. (A) Representative immunofluorescence images revealing activated (a) STAT3 and (b) P65 in colonic cancer tissue and precancerous tissue. Scale bar, $100 \mu \mathrm{m}$. (B) Representative western blotting revealing the expression of p-P65, P65, p-STAT3 and STAT3 in stages I, II and III colonic tumor tissues. GAPDH was used as a control. (C) Band intensities of western blotting for p-P65/P65 and p-STAT3/STAT3 in stage I, II and III tissues were analyzed. The data are reported as the mean \pm standard deviation of experiments ( $=4)$. ${ }^{* *} \mathrm{P}<0.01$, phosphorylation levels of P65 in stage I vs. in stage III; ${ }^{* *} \mathrm{P}<0.01$, phosphorylation levels of STAT3 in stage I tissues vs. in stage II, III tissues. STAT3, signal transducer and activator of transcription 3; p-, phosphorylated.

colorectal adenocarcinoma) and SW620 (ATCC ${ }^{\circledR}$ CCL-227 ${ }^{\mathrm{TM}}$, organism, human; tissue, colon; derived from metastatic site, lymph node; disease, colorectal adenocarcinoma) cells were purchased from the American Type Culture Collection (ATCC; Manassas, VA, USA). Three cell lines within passages 10 were used in all experiments. Three cell lines were maintained at $37^{\circ} \mathrm{C}$ in a humidified incubator containing $5 \% \mathrm{CO}_{2}$. The SW480 and SW620 cells were grown in Dulbecco's modified Eagle's medium (DMEM; Corning, Inc., Corning, NY, USA) supplemented with $10 \%$ fetal bovine serum (FBS; Gibco; Thermo Fisher Scientifc Inc., MA, USA). The HT29 cells were grown in McCoy's 5A medium (Corning, Inc.) supplemented with $10 \%$ FBS.

Cell proliferation and migration. The CCK- 8 kit was used to investigate SW480 cell proliferation. SW480 cells were plated in 96-well plates and grown for $72 \mathrm{~h}$. The procedure was performed according to the manufacturer's protocol. Subsequently, the mean percentages of cell survival and apoptosis were calculated. To inhibit STAT3 and NF- $\kappa \mathrm{B}$ signaling, $0.5,1$ and $2 \mu \mathrm{M}$ niclosamide (Selleck Chemicals, Houston, TX, USA) or 5, 15 and $30 \mu \mathrm{M} \mathrm{JSH}-23$ (Selleck Chemicals) was added to the cell culture medium. SW480 cell migration was investigated using Transwell assays. The bottom chambers were filled with medium with FBS, and the top chambers were seeded with medium without FBS containing $2 \times 10^{4}$ SW480 cells. The SW480 cells were allowed to migrate for $24 \mathrm{~h}$, and non-migrating cells were removed by scraping. The migratory cells were fixed with $100 \%$ methanol and stained with $0.05 \%$ crystal violet. The migratory cells were observed using a light microscope (Nikon Corp., Tokyo, Japan) and quantified by manual counting.

Statistical analysis. Statistical significance was assessed with two-way analysis of variance using GraphPad Prism software (version 5.0; GraphPad Software, Inc., La Jolla, CA, USA). $\mathrm{P}<0.05$ was considered to indicate a statistically significant difference. Where significance was revealed, comparisons were made using the Bonferroni post hoc test. All results are reported as the mean \pm standard deviation.

\section{Results}

Correlation of the expression of LYPD8 with STAT3/P65 phosphorylation and IL-6/TNF- $\alpha$ secretion in patients with $C R C$. The present study detected the expression and distribution of STAT3 and P65 in CRC tissues at different stages. As shown in Fig. 1A, using immunofluorescence experiments, increased expression levels of STAT3 and P65 were observed in stage II and IV CRC tissues compared with those in precancerous tissue. STAT3 and P65 were was expressed in the cytoplasm and nucleus. The results of the western blotting showed that the levels of p-P65/P65 and p-STAT3/STAT3 gradually increased between stage I and III (Fig. 1B and C). These results revealed increased phosphorylation levels of STAT3 and P65 in CRC tissues.

Previous studies have demonstrated that STAT3 and P65 can regulate the biological behavior of tumor cells and the 
A

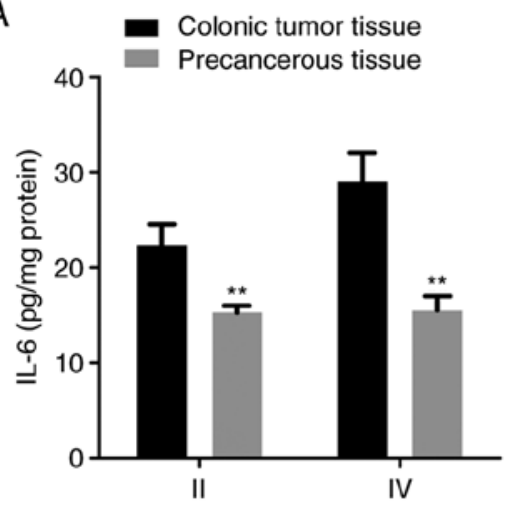

Secretion of IL-6

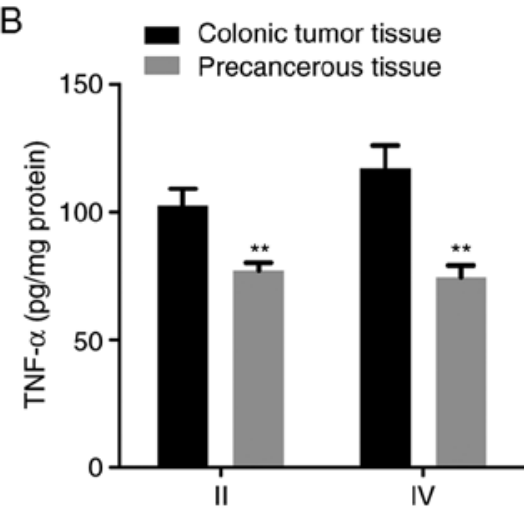

Secretion of TNF- $\alpha$

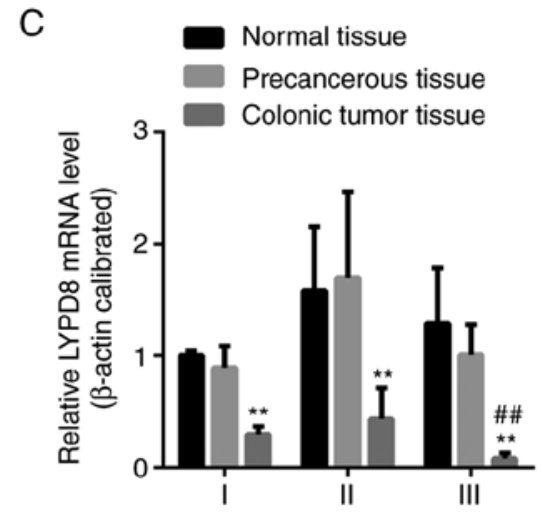

Expression of LYPD8

Figure 2. Association between IL-6/TNF- $\alpha$ and the expression of LYPD8 in colonic tumor tissue, precancerous tissue and normal tissue at different stages. (A) IL-6 and (B) TNF- $\alpha$ secretion were analyzed by ELISA in stage II and IV colonic tumor tissue and precancerous tissue. (C) Gene expression of LYPD8 in stage I, II and III colonic tumor tissue and precancerous tissue. $\beta$-actin was used as a control. The data are reported as the mean \pm standard deviation of experiments $(\mathrm{n}=6) .{ }^{* * *} \mathrm{P}<0.01$, LYPD8 mRNA expression of normal tissue, precancerous tissue vs. colonic tumor tissue in stage I, II and III tissues; ${ }^{\# \# /} \mathrm{P}<0.01$, LYPD8 mRNA expression of colonic tumor tissue in stage I tissues vs. in stage III tissues. LYPD8, Ly6/Plaur domain-containing 8; IL-6, interleukin-6; TNF- $\alpha$, tumor necrosis factor- $\alpha$.

expression of inflammatory mediators. Therefore, the present study detected the secretion of IL- 6 and TNF- $\alpha$ in CRC tissues at different stages. As shown in Fig. 2A and B, the secreted levels of IL- 6 and TNF- $\alpha$ in stage II and IV CRC tissues were significantly increased compared with those in corresponding precancerous tissues. These results revealed increased expression of IL- 6 and TNF- $\alpha$ in CRC tissues. Following this, the gene expression levels of LYPD8 in stage I, II and III CRC tissue; precancerous tissue; and normal tissue were assessed using RT-qPCR analysis (Fig. 2C). Compared with the precancerous tissue and normal tissue, the gene expression of LYPD8 was significantly reduced in stage I, II and III tissues. Furthermore, the expression of LYPD8 was reduced in stage III tissue compared with that in stage I tissue. Taken together, when STAT3/P65 is phosphorylated and IL-6/TNF- $\alpha$ is actively expressed, reduced expression of LYPD8 is noted in colonic cancer tissue at different stages.

Construction and overexpression of LYPD8 in CRC cells. The plasmid DNA for overexpressing LYPD8 was constructed using the eukaryotic expression vector (pIRES2), as shown in Fig. 3A and B, and the relative expression levels of LYPD8 in the HT29, SW480 and SW620 cells were examined by RT-qPCR analysis. The lowest expression of LYPD8 was noted in the SW480 cells, leading to selection of this cell line for the subsequent experiments. As shown in Fig. 3C, the gene expression of LYPD8 was significantly increased in the LYPD8 overexpression group (LYPD8 OE) compared with that in the empty pIRES2 group (control). These results indicated that the LYPD8 overexpression vector had been successfully used to induce the overexpression of LYPD8 in SW480 cells.

Subsequently, IL- 6 and TNF- $\alpha$ secretion were assessed in the LYPD8-overexpressing SW480 cells using ELISA. As shown in Fig. 3D, compared with the control group, IL-6 and TNF- $\alpha$ secretion were decreased in the LYPD8 OE group. This result revealed that the overexpression of LYPD8 inhibited the secretion of IL- 6 and TNF- $\alpha$ in SW480 cells. The protein expression levels of P65, p-P65, STAT3 and p-STAT3 in SW480 cells were then examined in the control,LYPD8 OE, LYPD8 OE + TNF- $\alpha$ and LYPD8 OE + IL- 6 groups, as demonstrated by western blotting. As shown in Fig. 3E and F, compared with the control group, the phosphorylation levels of p-P65/P65 and p-STAT3/STAT3 were significantly reduced in the LYPD8 OE group. However, this trend was restored when the SW480 cells were supplemented with TNF- $\alpha(5 \mathrm{ng} / \mathrm{ml})$ and IL-6 $(50 \mathrm{ng} / \mathrm{ml})$ in the culture medium. These results suggest that the overexpression of LYPD8 in SW480 cells induced the dephosphorylation of P65 and STAT3 and subsequently inhibited the secretion of TNF- $\alpha$ and IL-6.

Overexpression of LYPD8 inhibits CRC cell proliferation and migration. To investigate cell viability under the overexpression of LYPD8, CCK-8 assays were performed using SW480 cells. As shown in Fig. 4A, the overexpression of LYPD8 in SW480 cells inhibited cell viability compared with the control group. When the SW480 cells were supplemented with TNF- $\alpha(5 \mathrm{ng} / \mathrm{ml})$ and IL-6 $(50 \mathrm{ng} / \mathrm{ml})$ in the culture medium, the cell viability of the LYPD8 OE + TNF- $\alpha$ and LYPD8 OE+IL-6 groups was similar to that of the control group. This result indicated that the overexpression of LYPD8 inhibits SW480 cell viability, whereas TNF- $\alpha$ and IL-6 restore viability. Furthermore, it was found that niclosamide (STAT3 inhibitor) and JSH-23 (NF-кB inhibitor) also inhibit cell viability, similar to the overexpression of LYPD8 in SW480 cells (Fig. 4B). This result indicated that the overexpression of LYPD8 inhibited SW480 cell viability, and that this trend was similar to supplementation of STAT3 inhibitor and NF- $\mathrm{KB}$ inhibitor in the culture medium. Transwell assays were used to examine the effect of the overexpression of LYPD8 on SW480 cell migration. As shown in Fig. 4C and D, a more marked inhibitory effect on cell migration was observed in the LYPD8 OE group compared with that in the untreated cells group and control group. This result indicated that the overexpression of LYPD8 inhibits SW480 cell migration. 
A

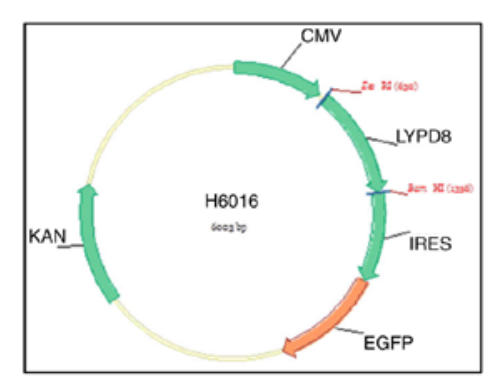

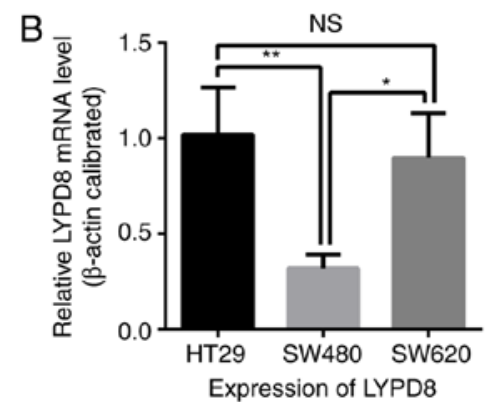
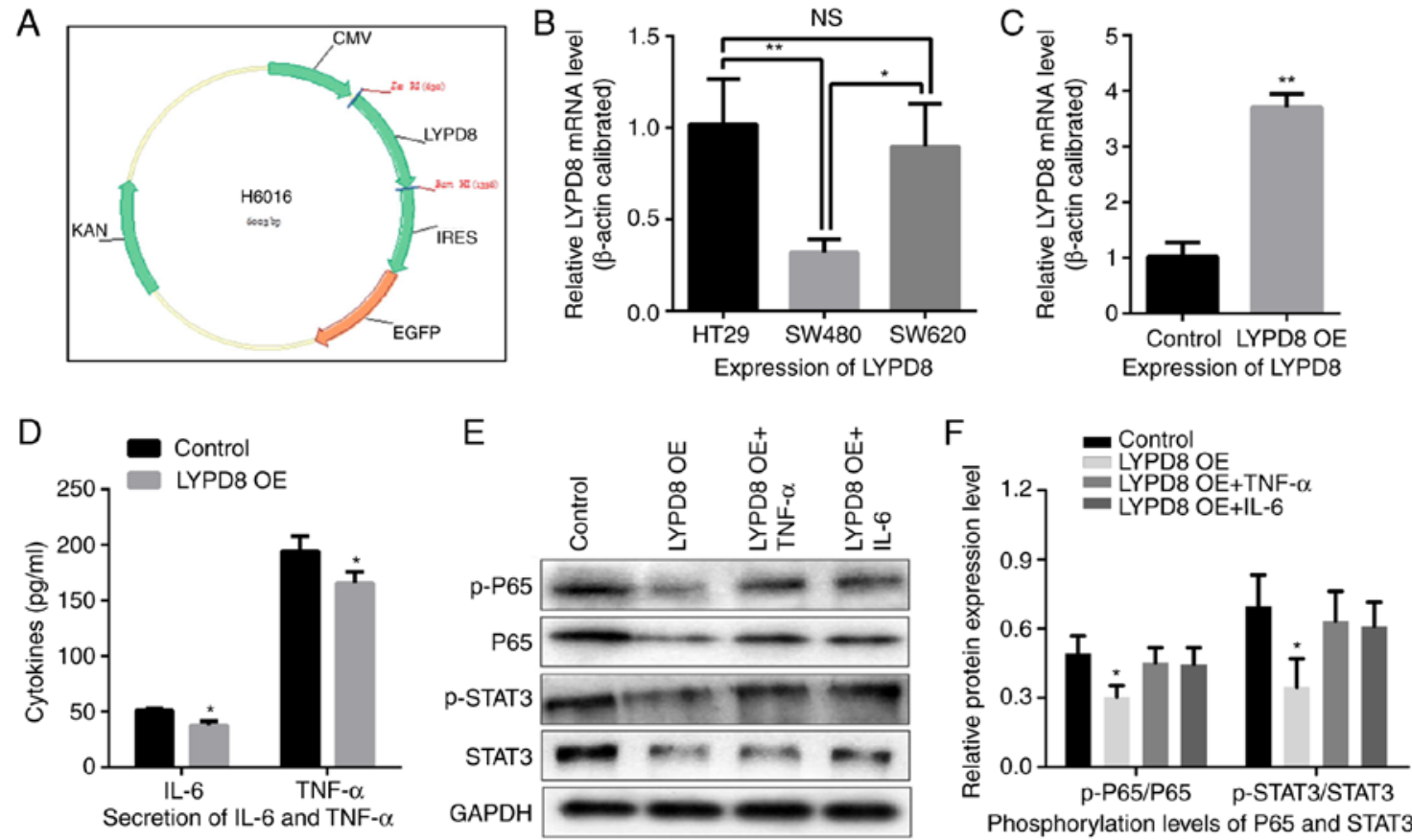

Phosphorylation levels of P65 and STAT3

Figure 3. Effects of the overexpression of LYPD8 on IL-6/TNF- $\alpha$ secretion and STAT3/P65 dephosphorylation in CRC cells. (A) cDNA coding for the LYPD8 gene was cloned into the eukaryotic expression vector. (B) Expression levels of LYPD8 in different CRC cells were determined by RT-qPCR analysis, using $\beta$-actin as a control. (C) Overexpression of LYPD8 via transient transfection into SW480 cells was confirmed by RT-qPCR analysis. (D) IL-6/TNF- $\alpha$ secretion was analyzed by ELISA in LYPD8 OE groups of SW480 cells. (E) Western blotting revealed expression levels of p-P65, P65, p-STAT3 and STAT3 in the control, LYPD8 OE, LYPD8 OE + TNF- $\alpha$ and LYPD8 OE + IL-6 groups of SW480 cells. GAPDH was used as a control. (F) Band intensities of western blotting for p-P65/P65 and p-STAT3/STAT3 in the control, LYPD8 OE, LYPD8 OE + TNF- $\alpha$ and LYPD8 OE + IL-6 groups were analyzed. The data are reported as the mean \pm standard deviation of experiments $(n=4) .{ }^{*} \mathrm{P}<0.05$, phosphorylation levels of P65 and STAT3 in LYPD8 OE groups vs. control, LYPD8 OE + TNF- $\alpha$ and LYPD8 OE + IL-6 groups; ${ }^{* *} \mathrm{P}<0.01$, expression of LYPD8 in control group vs. LYPD8 OE group. Control, empty pIRES2; LYPD8, Ly6/Plaur domain-containing 8; OE, overexpression; IL-6, interleukin-6; TNF- $\alpha$, tumor necrosis factor- $\alpha$; STAT3, signal transducer and activator of transcription 3; p-, phosphorylated; RT-qPCR, reverse transcription-quantitative polymerase chain reaction; NS, not significant.

A

A Effects of LYPD8 overexpression on cell proliferation

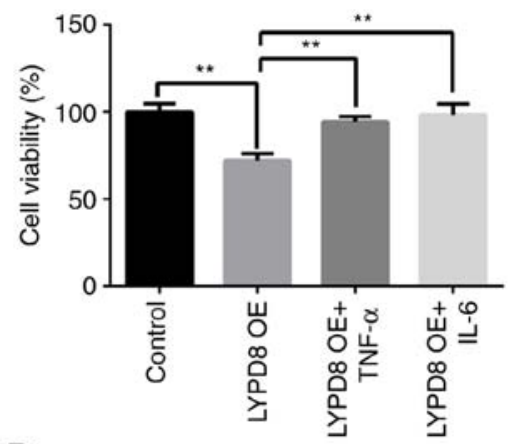

B

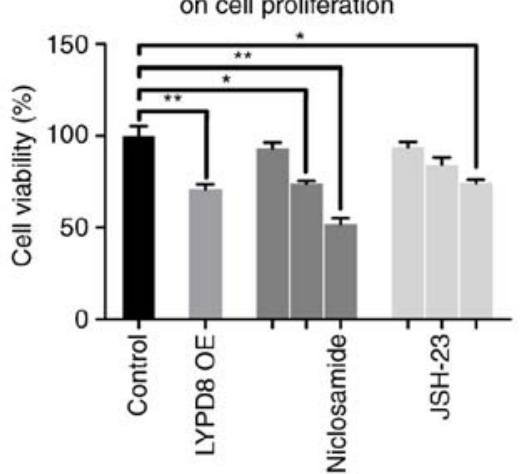

C
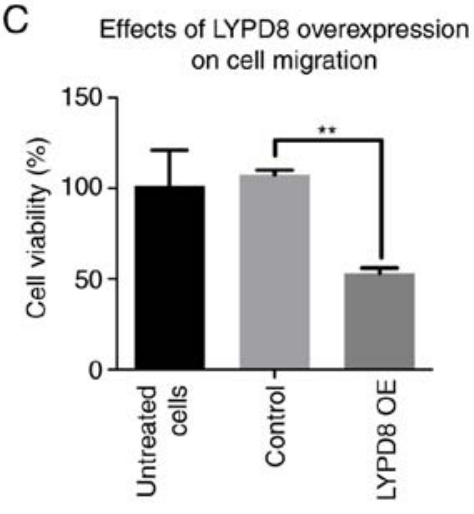

D

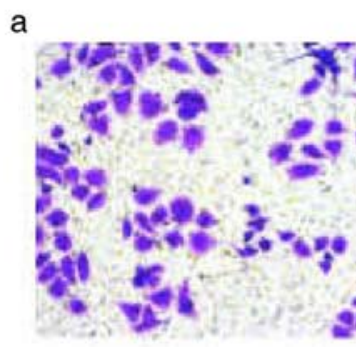

b

c

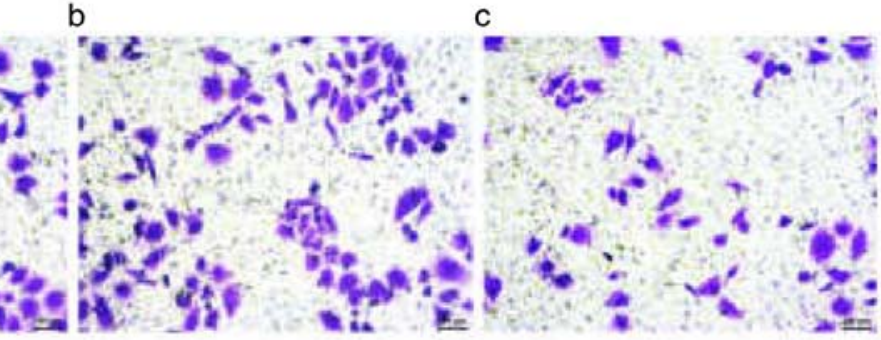

Untreated cells

Control

LYPD8 OE

Figure 4. Effects of the overexpression of LYPD8 on SW480 cell proliferation and migration. (A) Cell viability of the control,LYPD8 OE, LYPD8 OE + TNF- $\alpha$ and LYPD8 OE + IL-6 groups of SW480 cells. (B) SW480 cells were treated with different concentrations $(0.5,1$ and $2 \mu \mathrm{M})$ of niclosamide and different concentrations $(5,15$ and $30 \mu \mathrm{M})$ of JSH-23, respectively. (C) Numbers of migratory SW480 cells from the untreated, control and LYPD8 OE groups. (D) Transwell assay of SW480 cells from the (a) untreated, (b) control and (c) LYPD8 OE groups (magnification, x200). The data are reported as the mean \pm standard deviation of experiments $(\mathrm{n}=4) .{ }^{*} \mathrm{P}<0.05,{ }^{* *} \mathrm{P}<0.01$. Control, empty pIRES2 group; LYPD8, Ly6/Plaur domain-containing 8; OE, overexpression; IL-6, interleukin-6; TNF- $\alpha$, tumor necrosis factor- $\alpha$. 


\section{Discussion}

In colonic tumor tissue, a large number of inflammatory factors promote inflammation and increase incidence (19). An interactive network between cancer cells and the surrounding inflammatory microenvironment is formed, which promotes cancer cell growth, proliferation and migration (20). Inflammatory disorder induces the dysfunction of colon cancer-related oncogenes and tumor suppressor genes, which lead to tumorigenesis (21). Inflammatory reactions also lead to the progression and spread of CRC cells (22). LYPD8 is specifically expressed in colonic epithelial cells, whereas its expression is reduced in colonic epithelial cells from patients with UC (23). Mice lacking Lypd8 lack the bacteria-free space immediately above the epithelial layer of the colon, indicating that Lypd8 is critical for the segregation of intestinal bacteria and colonic epithelia $(1,3)$. Therefore, LYPD8 dysfunction is an important factor in the generation of colitis and an important regulatory protein in the development of colon cancer.

IL- 6 and TNF- $\alpha$ are important mediators involved in inflammatory reactions and serve an important regulatory role in cell behavior (24). IL-6 is a cytokine that is involved in the physiological and pathological processes of numerous types of cancer (25). Previous studies have reported that IL-6 is an important regulator in the progression and metastasis of certain tumors (26). TNF- $\alpha$ is secreted by macrophages or activated $\mathrm{T}$ cells and can promote cancer cell growth and metastasis (27). A high expression of TNF- $\alpha$ leads to disorderly association with other cytokines and is involved in disease mechanisms (28). The results of the present study demonstrated that the expression levels of IL- 6 and TNF- $\alpha$ in CRC tumor tissues were significantly increased compared with those in precancerous tissues, which is consistent with previous studies. The immune response is a regulatory response in the development of CRC involving inflammatory factors, including IL-6 and TNF- $\alpha$ (29). The results of the present study demonstrated that the overexpression of LYPD8 in CRC cells inhibited the secretion of IL-6 and TNF- $\alpha$.

IL-6 mediates signaling pathway transduction and the activation of transcriptional factors through the STAT3 signaling pathway to regulate tumor cell proliferation and migration (30). As STAT3 is required for the survival of intestinal epithelial cells and maintenance of mucosal integrity, excessive interference with systemic STAT3 activation can potentially cause gastrointestinal damage (31). When stimulated with extracellular cytokines, including IL-6, the tyrosine group of STAT3 is phosphorylated to p-STAT3, and homo or heterodimers form via the SH2 region. Following this, p-STAT3 dimers are transferred into the nucleus and induce the abnormal expression of downstream genes (32). In the present study, p-STAT3 was detected in SW480 cells of the control, LYPD8 OE, LYPD8 OE + TNF- $\alpha$ and LYPD8 OE + IL-6 groups, and the overexpression of LYPD8 reduced the phosphorylation of STAT3.

Previous studies have demonstrated that TNF- $\alpha$ upregulates NF- $\mathrm{kB}$ signaling pathway activity (33). NF- $\kappa \mathrm{B}$ is a protein that exhibits transcriptional function. NF- $\kappa \mathrm{B}$ initiates gene transcription involved in pathological processes with or without other cytokines (34). Due to the stimulating effect on tumor development by NF- $\kappa \mathrm{B}$, a series of drugs have been developed that target the activity of NF- $\mathrm{KB}(35)$. NF- $\mathrm{\kappa B}$ exists in cells is a dimeric state. P65/p50 heterodimers are the most common state, which are widely distributed in cells and are involved in the expression and regulation of multiple genes (36). The results of the present study demonstrated that the overexpression of LYPD8 promoted the phosphorylation of P65, leading to a change of cell behavior. In addition, the results demonstrated that the secretion of IL- 6 and TNF- $\alpha$ in CRC tissues was significantly increased compared with that in corresponding precancerous tissues, and the phosphorylation of STAT3/P65 was induced. By contrast, the expression of LYPD8 was significantly reduced in stage I, II, and III CRC tissues. These investigations remain in progress, and the aim of future investigations is to continue to identify the association between the protein expression of LYPD8 and the development of CRC.

In conclusion, the present study used clinical samples and revealed the association between LYPD8 and the expression of IL-6/TNF- $\alpha$. The mechanism by which LYPD8 is involved in regulating the STAT3 signaling pathway in CRC, and the effect of the overexpression of LYPD8 on CRC behaviors were examined. To the best of our knowledge, the present study is the first to examine the associations among LYPD8, inflammatory factors and the occurrence and development of CRC. To investigate the effect of LYPD8, the expression of LYPD8 was examined in clinical samples and then overexpressed in CRC cells. Previous studies had an important implication for examining the association between the inflammatory microenvironment and tumors. The present study provides a theoretical and experimental basis for the design of anti-inflammatory-targeted treatment for CRC.

\section{Acknowledgements}

Not applicable.

\section{Funding}

The present study was supported by the National Natural Science Foundation of China (grant nos. 81771502 and 81701820), the Natural Science Foundation of Zhejiang Province (grant no. LH19H160001) and the Medical Health Science and Technology Project of Zhejiang Provincial Health Commission (grant nos. 2018KY473, 2018PY025 and 2019KY737).

\section{Availability of data and materials}

The datasets generated during the present study are available from the corresponding author on reasonable request.

\section{Authors' contributions}

JX and JQ completed all trial procedures, the data analysis and were major contributors in writing the manuscript. WZhang was involved in the collection and processing of human cancer specimens. EC, GZ and GC guided the histopathological study of immunohistochemistry and all the methods and ideas of the cell experiments. FW and XS participated in the completion of the PCR and ELISA test procedures, and participated in the cultivation of cells. WZhou and ZS designed the overall idea of the experiment and provided theoretical guidance throughout 
the process, and participated in the revision of the manuscript and the processing of data. All authors read and approved the manuscript and agree to be accountable for all aspects of the research in ensuring that the accuracy or integrity of any part of the work are appropriately investigated and resolved.

\section{Ethics approval and consent to participate}

All experiments using human tissues were approved by the local institutional Ethics Committee (institutional review board reference no. 20140213-19). Written informed consent was obtained from patients prior to their inclusion in the study.

\section{Patient consent for publication}

Not applicable.

\section{Competing interests}

The authors confirm that they have no competing interests.

\section{References}

1. Okumura R, Kurakawa T, Nakano T, Kayama H, Kinoshita M, Motooka D, Gotoh K, Kimura T, Kamiyama N, Kusu T, et al: Lypd8 promotes the segregation of flagellated microbiota and colonic epithelia. Nature 532: 117-121, 2016.

2. Hsu C, Okumura R and Takeda K: Human LYPD8 protein inhibits motility of flagellated bacteria. Inflamm Regen 37: 23 , 2017.

3. Okumura R and Takeda K: Roles of intestinal epithelial cells in the maintenance of gut homeostasis. Exp Mol Med 49: e338, 2017.

4. Nishino K, Nishida A, Inoue R, Kawada Y, Ohno M, Sakai S, Inatomi O, Bamba S, Sugimoto M, Kawahara M, et al: Analysis of endoscopic brush samples identified mucosa-associated dysbiosis in inflammatory bowel disease. J Gastroenterol 53 : 95-106, 2018

5. Terzić J, Grivennikov S, Karin E and Karin M: Inflammation and colon cancer. Gastroenterology 138: 2101-2114, 2010.

6. Liu Y, Han ZP, Zhang SS, Jing YY, Bu XX, Wang CY, Sun K, Jiang GC, Zhao X, Li R, et al: Effects of inflammatory factors on mesenchymal stem cells and their role in the promotion of tumor angiogenesis in colon cancer. J Biol Chem 286: 25007-25015, 2011

7. Waldner $M$ and Neurath $M$ : Master regulator of intestinal disease: IL-6 in chronic inflammation and cancer development. Semin Immunol 26: 75-79, 2014.

8. Wu X, Huang C, He X, Tian YY, Zhou DX, He Y, Liu XH and $\mathrm{Li}$ J: Feedback regulation of telomerase reverse transcriptase: New insight into the evolving field of telomerase in cancer. Cell Signal 25: 2462-2468, 2013.

9. Oh $\mathrm{H}$ and Ghosh S: NF- $\mathrm{BB}$ : Roles and regulation in different CD4 ${ }^{+}$T-cell subsets. Immunol Rev 252: 41-51, 2013.

10. De Simone V, Franzè E, Ronchetti G, Colantoni A, Fantini MC, Di Fusco D, Sica GS, Sileri P, MacDonald TT, Pallone F, et al: Th17-type cytokines, IL- 6 and TNF- $\alpha$ synergistically activate STAT3 and NF- $\kappa$ B to promote colorectal cancer cell growth. Oncogene 34: 3493-3503, 2015.

11. Cortez M, Carmo LS, Rogero MM, Borelli P and Fock RA: A high-fat diet increases IL-1, IL- 6 , and TNF- $\alpha$ production by increasing NF- $\kappa \mathrm{B}$ and attenuating PPAR- $\gamma$ expression in bone marrow mesenchymal stem cells. Inflammation 36: 379-386, 2013.

12. Garbers C, Aparicio-Siegmund S and Rose-John S: The IL-6/gp130/STAT3 signaling axis: Recent advances towards specific inhibition. Curr Opin Immunol 34: 75-82, 2015.

13. Siveen KS, Sikka S, Surana R, Dai X, Zhang J, Kumar AP, Tan BK, Sethi G and Bishayee A: Targeting the STAT3 signaling pathway in cancer: Role of synthetic and natural inhibitors Biochim Biophys Acta 1845: 136-154, 2014.

14. Chen J, Wang J, Lin L, He L, Wu Y, Zhang L, Yi Z, Chen Y, Pang $X$ and Liu M: Inhibition of STAT3 signaling pathway by nitidine chloride suppressed the angiogenesis and growth of human gastric cancer. Mol Cancer Ther 11: 277-287, 2012.
15. Yu H, Lee H, Herrmann A, Buettner R and Jove R: Revisiting STAT3 signalling in cancer: New and unexpected biological functions. Nat Rev Cancer 14: 736-746, 2014

16. Huang C, Yang G, Jiang T, Zhu G, Li H and Qiu Z: The effects and mechanisms of blockage of STAT3 signaling pathway on IL-6 inducing EMT in human pancreatic cancer cells in vitro. Neoplasma 58: 396-405, 2011.

17. Xu J, Zhu C, Zhang Y, Jiang N, Li S, Su Z, Akaike T and Yang J: $\mathrm{hE}$-cadherin-Fc fusion protein coated surface enhances the adhesion and proliferation of human mesenchymal stem cells. Colloids Surf B Biointerfaces 109: 97-102, 2013.

18. Livak KJ and Schmittgen TD: Analysis of relative gene expression data using real-time quantitative PCR and the $2^{-\Delta \Delta C_{\mathrm{T}}}$ method. Methods 25: 402-408, 2001.

19. Gleeson M, Bishop NC, Stensel DJ, Lindley MR, Mastana SS and Nimmo MA: The anti-inflammatory effects of exercise: Mechanisms and implications for the prevention and treatment of disease. Nat Rev Immunol 11: 607-615, 2011.

20. Elinav E, Nowarski R, Thaiss C, Hu B, Jin C and Flavell RA: Inflammation-induced cancer: Crosstalk between tumours, immune cells and microorganisms. Nat Rev Cancer 13: 759-771, 2013.

21. Balkwill FR and Mantovani A: Cancer-related inflammation: Common themes and therapeutic opportunities. Semin Cancer Biol 22: 33-40, 2012.

22. Erreni M, Mantovani A and Allavena P: Tumor-associated macrophages (TAM) and inflammation in colorectal cancer. Cancer Microenviron 4: 141-154, 2011.

23. Allaire JM, Morampudi V, Crowley SM, Stahl M, Yu H, Bhullar K, Knodler LA, Bressler B, Jacobson K and Vallance BA: Frontline defenders: Goblet cell mediators dictate host-microbe interactions in the intestinal tract during health and disease. Am J Physiol Gastrointest Liver Physiol 314: G360-G377, 2018.

24. Popko K, Gorska E, Stelmaszczyk-Emmel A, Plywaczewski R, Stoklosa A, Gorecka D, Pyrzak B and Demkow U: Proinflammatory cytokines Il- 6 and TNF- $\alpha$ and the development of inflammation in obese subjects. Eur J Med Res 15 (Suppl) S120-S122, 2010.

25. Mihara M, Hashizume M, Yoshida H, Suzuki M and Shiina M: IL-6/IL-6 receptor system and its role in physiological and pathological conditions. Clin Sci 122: 143-159, 2012.

26. McAllister SS and Weinberg RA: The tumour-induced systemic environment as a critical regulator of cancer progression and metastasis. Nat Cell Biol 16: 717-727, 2014.

27. Guan YQ, Li Z and Liu JM: Death signal transduction induced by co-immobilized TNF- $\alpha$ plus IFN- $\gamma$ and the development of polymeric anti-cancer drugs. Biomaterials 31: 9074-9085, 2010.

28. Emi Aikawa N, de Carvalho JF, Artur Almeida Silva $\mathrm{C}$ and Bonfá E: Immunogenicity of anti-TNF-alpha agents in autoimmune diseases. Clin Rev Allergy Immunol 38: 82-89, 2010.

29. Zamarron BF and Chen W: Dual roles of immune cells and their factors in cancer development and progression. Int J Biol Sci 7: 651-658, 2011.

30. Guo Y, Xu F, Lu T, Duan Z and Zhang Z: Interleukin-6 signaling pathway in targeted therapy for cancer. Cancer Treat Rev 38: 904-910, 2012.

31. Neurath MF and Finotto S: IL-6 signaling in autoimmunity, chronic inflammation and inflammation-associated cancer. Cytokine Growth Factor Rev 22: 83-89, 2011.

32. Lin L, Hutzen B, Zuo M, Ball S, Deangelis S, Foust E, Pandit B, Ihnat MA, Shenoy SS, Kulp S, et al: Novel STAT3 phosphorylation inhibitors exhibit potent growth-suppressive activity in pancreatic and breast cancer cells. Cancer Res 70: 2445-2454, 2010.

33. Lü L, Tang D, Wang L, Huang LQ, Jiang GS, Xiao XY and Zeng FQ: Gambogic acid inhibits TNF- $\alpha$-induced invasion of human prostate cancer PC3 cells in vitro through PI3K/Akt and NF- $\kappa$ B signaling pathways. Acta Pharmacol Sin 33: 531-541, 2012.

34. Ben-Neriah Y and Karin M: Inflammation meets cancer, with $\mathrm{NF}-\kappa \mathrm{B}$ as the matchmaker. Nat Immunol 12: 715-723, 2011.

35. Miller S, Huang R, Sakamuru S, Shukla SJ, Attene-Ramos MS, Shinn P, Van Leer D, Leister W, Austin CP, Xia M, et al: Identification of known drugs that act as inhibitors of NF-kappaB signaling and their mechanism of action. Biochem Pharmacol 79: 1272-1280, 2010.

36. Wan F and Lenardo MJ: The nuclear signaling of NF-kappaB: Current knowledge, new insights, and future perspectives. Cell Res 20: 24-33, 2010. 